R. Pail*, J. Bamber, R. Biancale, R. Bingham, C. Braitenberg, A. Eicker, F. Flechtner, T. Gruber, A. Güntner, G. Heinzel, M. Horwath, L. Longuevergne, J. Müller, I. Panet, H. Savenije, S. Seneviratne, N. Sneeuw, T. van Dam, and B. Wouters

\title{
Mass variation observing system by high low inter-satellite links (MOBILE) - a new concept for sustained observation of mass transport from space
}

DOI: https://doi.org/10.1515/jogs-2019-0006

Received October 5, 2018; accepted March 10, 2019

*Corresponding Author: R. Pail: Technical University of Munich, Institute of Astronomical and Physical Geodesy, Munich, Germany, E-mail: roland.pail@tum.de

J. Bamber: University of Bristol, School of Geographical Sciences, Bristol, UK

R. Biancale: Centre National d'Études Spatiales (CNES), Toulouse, France

R. Bingham: University of Bristol, School of Geographical Sciences, Bristol, UK

C. Braitenberg: University of Trieste, Dptm. of Mathematics \& Earth Sciences, Trieste, Italy

A. Eicker: HafenCity Univ. Hamburg, Geodesy and Adjustment Theory, Hamburg, Germany

F. Flechtner: GFZ \& Technische Univ. Berlin, Chair of Physical Geodesy, Berlin, Germany

T. Gruber: Technical University of Munich, Institute of Astronomical and Physical Geodesy, Munich, Germany

A. Güntner: Deutsches GeoForschungsZentrum Potsdam, Sect. Hydrology, Potsdam, Germany

G. Heinzel: AEl, Max Planck Inst. for Gravitational Physics, Hannover, Germany

M. Horwath: Technische Universität Dresden, Inst. of Planetary Geodesy, Dresden, Germany

L. Longuevergne: Université Rennes, CNRS, Géosciences, Rennes, France

J. Müller: Leibniz Universität Hannover, Institute of Geodesy, Hannover, Germany

I. Panet: Institut National de l'Information Géographique et Forestière, Paris, France

H. Savenije: Technical University Delft, Water Resources Section, Delft, Netherlands

S. Seneviratne: ETH Zürich, Institute for Atmospheric and Climate Science, Zürich, Switzerland

N. Sneeuw: Universität Stuttgart, Institute of Geodesy, Stuttgart, Germany

T. van Dam: University of Luxembourg, FSTC, Luxembourg

B. Wouters: Univ. Utrecht, Institute for Marine and Atmospheric Research, Utrecht, Netherlands
Abstract: As changes in gravity are directly related to mass variability, satellite missions observing the Earth's time varying gravity field are a unique tool for observing mass transport processes in the Earth system, such as the water cycle, rapid changes in the cryosphere, oceans, and solid Earth processes, on a global scale. The observation of Earth's gravity field was successfully performed by the GRACE and GOCE satellite missions, and will be continued by the GRACE Follow-On mission. A comprehensive team of European scientists proposed the next-generation gravity field mission MOBILE in response to the European Space Agency (ESA) call for a Core Mission in the frame of Earth Explorer 10 (EE10). MOBILE is based on the innovative observational concept of a high-low tracking formation with micrometer ranging accuracy, complemented by new instrument concepts. Since a high-low tracking mission primarily observes the radial component of gravity-induced orbit perturbations, the error structure is close to isotropic. This geometry significantly reduces artefacts of previous along-track ranging low-low formations (GRACE, GRACE-Follow-On) such as the typical striping patterns. The minimum configuration consists of at least two medium-Earth orbiters (MEOs) at $10000 \mathrm{~km}$ altitude or higher, and one low-Earth orbiter (LEO) at 350$400 \mathrm{~km}$. The main instrument is a laser-based distance or distance change measurement system, which is placed at the LEO. The MEOs are equipped either with passive reflectors or transponders. In a numerical closed-loop simulation, it was demonstrated that this minimum configuration is in agreement with the threshold science requirements of $5 \mathrm{~mm}$ equivalent water height (EWH) accuracy at $400 \mathrm{~km}$ wavelength, and $10 \mathrm{~cm}$ EWH at $200 \mathrm{~km}$. MOBILE provides promising potential future perspectives by linking the concept to existing space infrastructure such as Galileo nextgeneration, as future element of the Copernicus/Sentinel programme, and holds the potential of miniaturization 
even up to swarm configurations. As such MOBILE can be considered as a precursor and role model for a sustained mass transport observing system from space.

Keywords: mass transport - next-generation gravity mission - gravity field - high-low tracking - spherical harmonics

\section{Introduction}

Climate change is one of the biggest societal challenges today. The underlying processes are most frequently related to mass variations in the Earth system. Mass is redistributed within the Earth system over a full range of spatial and temporal scales. As mass variations directly induce changes of the Earth gravity field, satellite missions observing the Earth's time varying gravity field are a unique tool for observing mass redistribution in the Earth system, such as the terrestrial water cycle, variations in the cryosphere and oceans, on a global scale. The dedicated gravity mission Gravity Recovery And Climate Experiment (GRACE) (Tapley et al. 2014) provided the first global determination of Earth's mass transport by measuring spatial and temporal changes in the gravity field caused by mass variations at all depths, while the static gravity field retrieved from the Gravity field and steady-state Ocean Circulation Explorer (GOCE) mission (Drinkwater et al. 2013) has improved our knowledge of the long-term mass distribution and has provided the physical reference surface of the geoid with a resolution down to $70-80 \mathrm{~km}$.

This is key information, as direct observations of mass variations and mass transport are difficult to obtain. For example, almost no direct observations of evapotranspiration are available. Storage changes in deep soil layers and groundwater are mostly inaccessible to conventional observation techniques. Current estimates for soil moisture derived from remote sensing satellites are usually based on the measurement of the uppermost few centimeters of soil, depending on the penetration depth, leaving the largest part of the water column unexplored. The same holds for the deep ocean circulation, an essential but hidden part of the climate system, playing an important role in heat transport and carbon dioxide sequestration. Additional limitations to a closure of the global water balance are imposed by the scarcity of data on freshwater run-off, limitations of satellite altimetry along coasts and ice sheet margins, and in high latitudes.

A resolution adopted by the Council of the International Union of Geodesy and Geophysics (IUGG 2015) calls for sustained observing of mass transport processes from space (Pail et al. 2015a). Understanding how extreme episodic events such as droughts, floods, earthquakes and volcanos, may relate to the longer-term evolution of the water-cycle or stress build-up, or resolving the fluxes at the boundaries between Earth system components, requires high accuracy time-varying gravity coverage over a full range of spatial and temporal scales that is currently unavailable. Only by mapping time varying gravity with a substantially increased spatial resolution, we can fully understand how changes in the Earth system develop across scales, how they modify the water fluxes, and how they are related to human activities. The understanding we may achieve is needed to address challenges facing human societies for a sustainable development over the short and long term. They include understanding sea level rise, and assessing the changing water availability for freshwater supply, agriculture and industry, especially in regions where freshwater resources are under severe threat, and anthropogenic demands and stresses are expected to further increase in the decades to come. A minimum of 30 years of observation is necessary to disentangle anthropogenic impact from natural variability (GCOS 2016).

The big success of the GRACE mission led to the implementation of GRACE Follow-On (GRACE-FO) (Flechtner et al. 2016), which was successfully launched in May 2018 and will continue the 15 years of mass transport time series obtained from GRACE. Recently, the United States National Academies of Sciences, Engineering, and Medicine published the decadal strategy for Earth observation from space (NAS 2018), where mass change was identified as one of the top 5 observables to be implemented by future US Earth observation missions in order to ensure continuity and enable long-term mass budget analyses of the Earth system. Again it is proposed to plan for a singlepair mission, which should follow GRACE-FO. This clearly demonstrates the importance of sustained gravity monitoring from space.

On European side, during the last couple of years several attempts have been made by the gravity-related science community to propose a next-generation gravity field mission as an Earth Explorer mission to the European Space Agency (ESA). The proposed mission constellations differed from the typical GRACE-type concept of an in-line pair, with which only the along-track component of the Earth's gravity field can be observed, leading to a very anisotropic error structure and the typical striping patterns in the resulting temporal gravity solutions. In 2010 the mission proposal "e.motion - Earth System Mass Transport Mission" (Panet et al. 2012) was submitted in response to ESA's Earth Explorer 8 call. It was based on a satellite pair in pendulum configuration, where the second satellite performs a pendulum motion with respect to 
the leading satellite, thus observing not only the alongtrack, but also parts of the cross-track component. In 2016 e.motion ${ }^{2}$ (Gruber et al. 2015) was proposed as ESA Earth Explorer 9 mission. In the basic configuration, it contained one out of two in-line pairs of a so-called Bender doublepair configuration (Bender et al. 2008). This set-up of a polar and an inclined pair with an inclination of 65-70 degrees again results in a significantly improved isotropy and reduction of stripes (Daras and Pail 2017).

In this paper we present an innovative observation concept of high-precision high-low inter-satellite ranging, which was proposed as the MOBILE mission in response to ESA's Earth Explorer 10 call. In Section 2 the science and mission objectives are outline. In Section 3 the technical concept, including the observation constellation and the key payload, is presented. The achievable performance with this mission concept is quantified by means of a numerical closed-loop simulation in Section 4. Finally, in Chapter 5 the main conclusions are drawn, and an outlook to future perspectives is given.

\section{Science Objectives and Requirements}

\subsection{Science Objectives}

The science objectives and requirements of MOBILE mainly follow the science and user needs as described in (Pail et al. 2015a). The following main science objectives have been identified:

1. Estimating continental water storage and freshwater fluxes

2. Quantification of large-scale flood and drought events and their monitoring and forecasting

3. Understanding mass balance of ice sheets and large glacier systems

4. Deciphering sea level variations and changes in ocean transport

5. Validation and assimilation into global climate models

6. Connecting solid Earth near surface changes to internal dynamics

7. Process couplings and contribution to a consistent Earth system model

8. Provision of time-variable gravity reference for the Global Geodetic Reference Frame (GGRF)

With these objectives, MOBILE contributes to an improved understanding of different components of the Earth sys- tem, and addresses a number of Essential Climate Variables (ECV; (GCOS 2016)) such as groundwater, snow cover, soil moisture, ice sheets, glaciers and ice caps, sea level, surface currents, and sub-surface currents. Regarding item number 8 , MOBILE would mainly provide information about the temporal evolution of physical reference frames (for example in regions with strong vertical motion such as glacial isostatic adjustment effects in Fennoscandia or Canada), while it would hardly be able to compete with gradiometer missions such as GOCE regarding the achievable spatial resolution of the static gravity field.

The relevant spatial and temporal scales for achieving these research objectives, as well as the amplitude of the signals, are described in Fig. 1. An accuracy at the level of $10 \%$ of these signal amplitudes would allow significant advances in our understanding of Earth's mass transport. When appropriate, these specifications are converted into equivalent temporal variations of the geoid and gravity. Conversion depends on spatial scales, or spherical harmonic (SH) degrees. E.g., a mass variation of $1 \mathrm{~cm}$ equivalent water height (EWH) in a spherical cap of radius $2000 \mathrm{~km}(800 \mathrm{~km}, 400 \mathrm{~km}, 200 \mathrm{~km}, 100 \mathrm{~km})$ maps to a $0.5 \mathrm{~mm}$ amplitude geoid variation $(0.3 \mathrm{~mm}, 0.15 \mathrm{~mm}$, $0.08 \mathrm{~mm}, 0.04 \mathrm{~mm}$, respectively).

\subsection{Science requirements and user needs}

As the mass signals related to the science objectives cover a range of spatial and temporal scales, in accordance with Pail et al. (2015b). and IGSWG (2016) a threshold scenario is defined, which allows us to make a significant advance with respect to our current knowledge, and a target scenario, which means a breakthrough in the way we use gravity data in Earth mass transport monitoring. Specifically, the science requirements for MOBILE are:

(1) To recover temporal gravity and mass variations in the Earth system with a $150 \mathrm{~km}$ spatial resolution or better, with a global coverage;

(2) To recover small amplitude mass variations with an approximately 5 times (threshold) to 50 times (target) increased sensitivity as compared to current knowledge;

(3) To resolve mass variations at daily to decadal time scales. While the high temporal resolution results from the enhanced data quality, the long timescales are obtained by extending the existing satellite gravity records by a time series of at least 5-7 years.

The signal characteristics have been studied in detail by Pail et al. (2015b), cf. also Fig. 1. Hence most estimates in this section are based on this reference. A higher spatial resolution than currently achievable is needed in all fields 


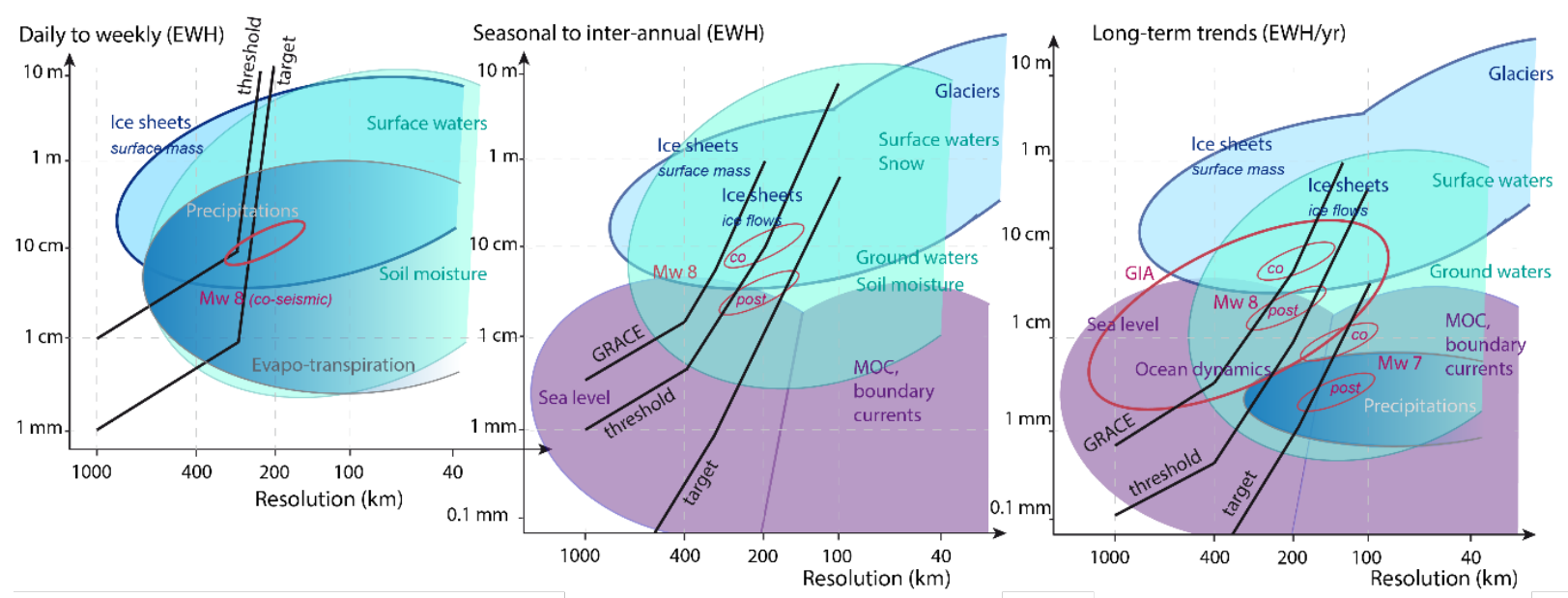

Fig. 1. Signal amplitudes of mass variations in equivalent water height (EWH) as a function of spatial resolution, together with presentday accuracy and resolution (GRACE) and with MOBILE threshold and target performances. Solid Earth mass variations are converted to EWH. Contributions from daily to weekly variations (left panel), from seasonal to inter-annual variations (middle panel), and from long-term trends (right panel). Drawn from (Pail et al. 2015b).

of application. For continental water storage monitoring, $10 \%$ of the river basins worldwide have characteristic sizes larger than $550 \mathrm{~km}, 30 \%$ of the basins have sizes between 550 and $330 \mathrm{~km}$, and $45 \%$ between 330 and $180 \mathrm{~km}$, while the size of global hydrological modelling grid cells can be of the order of $50 \mathrm{~km}$. An increase in spatial resolution down to $150 \mathrm{~km}$ would allow for the monitoring of groundwater depletion in smaller scale hot spots such as many European hydrological catchments. The finer spatial resolution would also allow for the recovery of mass variations in elongated basins such as the Rhine or Danube catchments. In a similar fashion, such an increase in spatial resolution would more than double the number of drainage basins within ice sheets that could be resolved.

Many mass signals in the water cycle and within the solid Earth are small in amplitude, hence demand a high accuracy. The MOBILE threshold scenario will allow significant advances in determining regional ocean mass variations, processes associated with large earthquakes $(\mathrm{Mw}$ 7.8 and above) or in separating large glacier complexes and monitoring medium-size hydrological basins with a $1.5 \mathrm{~cm}$ EWH precision. The MOBILE target scenario will provide a leap into signal separation and leakage reduction from neighbouring mass sources. For instance, the glacial isostatic adjustment (GIA) signal over Antarctica leaks by about $200 \mathrm{~km}$ into the oceans, with a $1 \mathrm{~cm} \mathrm{EWH/yr}$ magnitude (Pail et al. 2015a, Pail et al. 2015b). The target scenario would allow for the separation of this signal from the ice mass variations. The high accuracy would also enable us to resolve the small ocean circulation signals, such as inter-annual variations of the Atlantic Meridional Overturning Circulation (AMOC).

Just the extension of the time series beyond GRACEFO will allow us to understand how anthropogenic forcings interact with climate changes in the changing water cycle, and the higher spatial resolution resulting from MOBILE will improve the water cycle versus solid Earth signal separation. By providing long time series of mass variations, MOBILE, as a precursor of a sustained mass transport observing system from space, will thus also enhance the value of data from preceding missions.

The analysis of the signal characteristics enables us to formulate, in accordance with (Pail et al. 2015a), the science requirements for MOBILE threshold and target scenarios, which are summarized in Table 1, and graphically illustrated in Fig. 1.

\section{Technical concepts}

\subsection{Observation Concept}

In order to achieve the science objectives as specified in Section 2.1, MOBILE consists of a constellation of high and low orbiting satellites. In such a constellation, the main observable is the inter-satellite distance between pairs of satellites (as done for GRACE and GRACE Follow-On). However, instead of observing gravity-induced inter-satellite distance changes between two low orbiting satellites in a pearl-string configuration, distance variations between 
Table 1. MOBILE threshold and target science requirements and user needs.

\begin{tabular}{|c|c|c|c|c|}
\hline \multirow{2}{*}{ Spatial Resolution } & \multicolumn{2}{|c|}{ Equivalent Water Height } & \multicolumn{2}{|c|}{ Geoid } \\
\hline & Monthly field & Long-term trend & Monthly field & Long-term trend \\
\hline \multicolumn{5}{|c|}{ Threshold requirements } \\
\hline $400 \mathrm{~km}$ & $5 \mathrm{~mm}$ & $0.5 \mathrm{~mm} / \mathrm{yr}$ & $50 \mu \mathrm{m}$ & $5 \mu \mathrm{m} / \mathrm{yr}$ \\
\hline $200 \mathrm{~km}$ & $10 \mathrm{~cm}$ & $1 \mathrm{~cm} / \mathrm{yr}$ & $0.5 \mathrm{~mm}$ & $0.05 \mathrm{~mm} / \mathrm{yr}$ \\
\hline $150 \mathrm{~km}$ & $50 \mathrm{~cm}$ & $5 \mathrm{~cm} / \mathrm{yr}$ & $1 \mathrm{~mm}$ & $0.1 \mathrm{~mm} / \mathrm{yr}$ \\
\hline \multicolumn{5}{|l|}{ Target requirements } \\
\hline $400 \mathrm{~km}$ & $0.5 \mathrm{~mm}$ & $0.05 \mathrm{~mm} / \mathrm{yr}$ & $5 \mu \mathrm{m}$ & $0.5 \mu \mathrm{m} / \mathrm{yr}$ \\
\hline $200 \mathrm{~km}$ & $1 \mathrm{~cm}$ & $0.1 \mathrm{~cm} / \mathrm{yr}$ & $0.05 \mathrm{~mm}$ & $5 \mu \mathrm{m} / \mathrm{yr}$ \\
\hline $150 \mathrm{~km}$ & $5 \mathrm{~cm}$ & $0.5 \mathrm{~cm} / \mathrm{yr}$ & $0.1 \mathrm{~mm}$ & $0.01 \mathrm{~mm} / \mathrm{yr}$ \\
\hline
\end{tabular}

medium (MEO; several thousand $\mathrm{km}$ ) and low orbiting satellites (LEO; several hundred $\mathrm{km}$ ) are observed. As second gravity observation type, high-precision orbit positions based on GNSS orbit determination are used. This idea of high-precision high-low tracking was first investigated for geostationary and GNSS satellites, and the main error sources and the corresponding achievable performance was analysed in (Hauk et al. 2017). However, in order to present a stand-alone concept without the need to place additional payload to other space infrastructure, for the MOBILE proposal, dedicated MEO satellites were included in the concept.

The minimum configuration consists of at least two MEOs (e.g. $10000 \mathrm{~km}$ or higher) and one LEO (around 350$400 \mathrm{~km}$ ) in the same orbital plane. The two MEOs are separated by 180-degree mean anomaly in order to maximize the observation time. Figure 2 shows a schematic overview of this minimum satellite configuration.

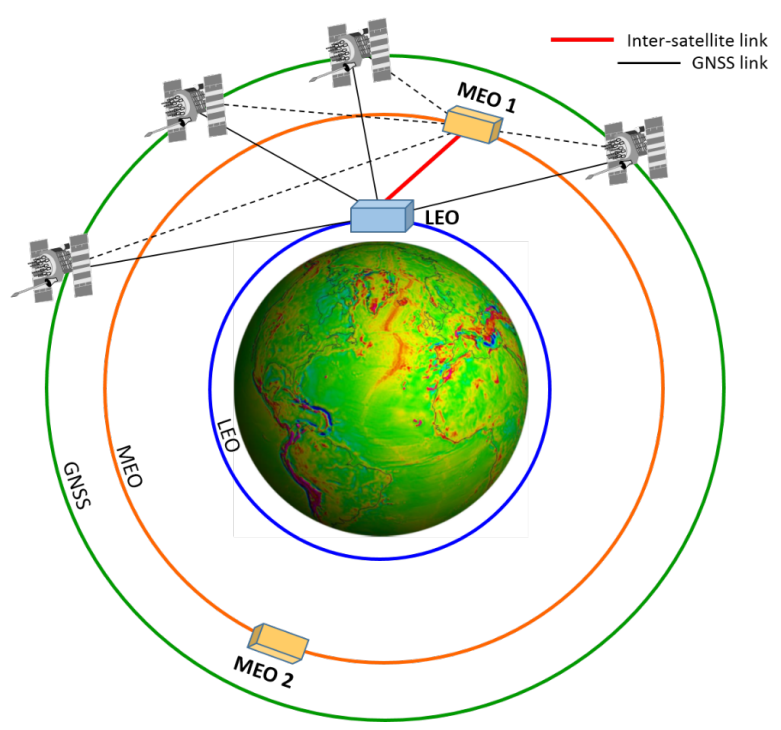

Fig. 2. MOBILE mission constellation: high-precision inter-satellite links between LEO and MEOs (red), micro-wave links from GNSS satellites to MEOs and LEO (black).
All three satellites fly in a polar orbit in order to maintain a long-term stable formation (no relative drifts of the orbit planes). The main observable are range measurements from the LEO to the MEOs, where the MEOs are alternating targets. The main instrument shall be a laser-based distance or distance change measurement system, which will be placed at the LEO. The MEOs will be equipped with passive reflectors or transponders. The target ranging accuracy is on the micrometer level. It shall be emphasized that in this concept the LEO satellite will be equipped with the active instrumentation, also including directiondepending pointing capabilities. The more sophisticated design of the LEO satellite has to be done anyway to keep the satellite in a low Earth orbit. In contrast, the design of the MEO satellite can be quite simple, and ideally its payload is only of passive nature.

On top of this minimum scenario, optionally the implementation of a third or fourth MEO satellite could be considered to further increase the mission performance. In this case the 3 to 4 MEO satellites will be separated by 120 or 90 -degree mean anomaly, respectively. In principle, also a second LEO could be considered. However, since the upper cost limit of the EE10 call was defined as 225 million Euros purely for the satellite(s), it is likely that a second more complex LEO satellite would not fit into the cost frame.

The orbit selection of the MOBILE constellation needs to be carefully done in order to ensure that the science goals can be achieved. In particular the orbit altitude of the LEO satellites drives the sensitivity to the Earth gravity field, and consequently defines the upper limit of spatial resolution to be achieved. Figure 3 shows results for a MOBILE constellation in dependence of the altitude of the LEO satellite. It identifies for example that by lowering the orbit from 400 to $346 \mathrm{~km}$ one can reach a gain in spatial resolution at a level of about $30 \mathrm{~km}$ (from SH degree 75 to 85 corresponding to spatial resolution $267 \mathrm{~km}$ to $235 \mathrm{~km}$ ). The curves in Fig. 3 are expressed in terms degree RMS of 
equivalent water heights (EWH), which is computed from fully normalized coefficients of a spherical harmonic series expansion $\left\{\bar{C}_{n m}, \bar{S}_{n m}\right\}$ of degree $n$ and order $m$, by

$$
\sigma_{n}(E W H)=\frac{a \rho_{e}}{3 \rho_{w}} \frac{2 n+1}{1+k_{n}} \sqrt{\sum_{m=0}^{n} \bar{C}_{n m}^{2}+\bar{S}_{n m}^{2}},
$$

where $\rho_{w}$ and $\rho_{e}$ represent the average densities of water and Earth, $a$ the semi-major axis of the Earth, and $k_{n}$ the load Love number of degree $n$.

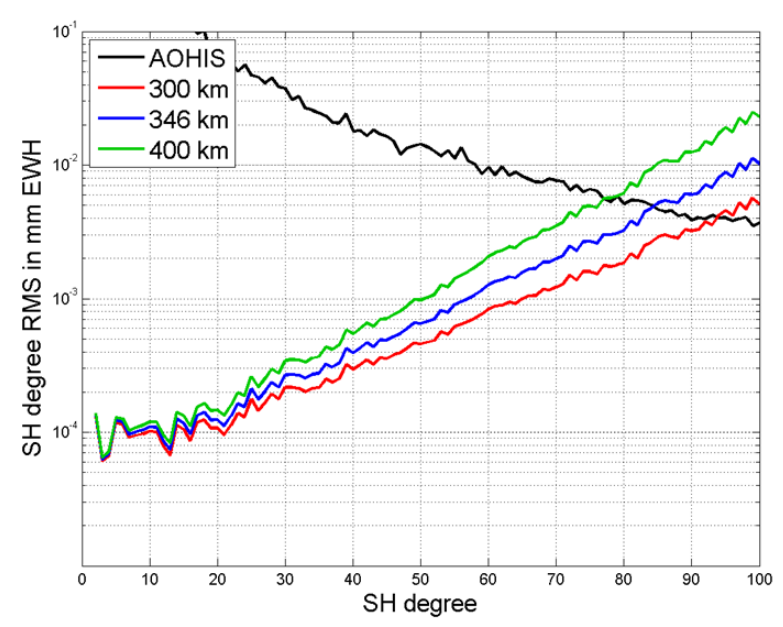

Fig. 3. Residual errors of monthly gravity field recovery compared to simulated signal (black curve) including only instrument errors, showing the impact of orbit height for MOBILE baseline configuration. The blue curve is equivalent to Figure 5 .

Another important issue related to the choice of optimal orbit heights is the reduction of temporal aliasing due to under-sampling. In (Murböck et al. 2015) a number of optimal orbit altitude bands are identified, where the impact of temporal aliasing is significantly reduced. Several numerical simulations have shown, that regarding orbit choice and related ground track coverage there is some design freedom, provided that the repeat period of the LEOs is long enough to guarantee sufficient spatial resolution and avoiding spatial undersampling. Regarding temporal resolution, the target consists in computing weekly to monthly gravity field solutions. However, the "Wiese parameterization" (Wiese et al. 2011) as described in Section 4 will facilitate to estimate long-wavelength gravity fields also on a daily or 2-daily basis up to SH degree 15 to 20 , thus enabling short latencies for service applications.

The main advantage of the high-low tracking concept compared to the low-low pearl-string configuration implemented in GRACE and GRACE-FO is, that multi-directional distance variations, with a strong dominance of the radial component, will be observed. The resulting (close to) isotropic error behaviour of this concept will reduce significantly the striping patterns caused by having only onedirectional observations, which is one of the key limiting factors of nowadays temporal gravity solutions, and inherently will improve the spatial resolution. Therefore, substantial post-filtering strategies to reduce these numerical artefacts can be largely avoided. The impact of the high-low observation geometry is nicely visible in Fig. 4. The triangles in the left column show the formal errors for the MOBILE high-low tracking concept (bottom), and in comparison GRACE/GRACE-FO low-low tracking (top), demonstrating that the first concept has an almost uniform (isotropic) error spectrum. A perfect isotropic behaviour implies that the colour of the triangle in the left column depends on the degree only, but there is no variation within the orders of a respective degree. The improvement in isotropy is even better visible when analysing the spatial signatures of the covariance function of the gravitational potential, which are displayed in the middle and right columns of Fig. 4. Shown is the covariance between two points. One of them is kept fixed at Greenwich meridian (geographical longitude $\lambda=0^{\circ}$ ) and either at the equator with geographical latitude $\varphi=0^{\circ}$ (middle column) or at a latitude of $\varphi=45^{\circ}$ (right column), while the other one is varying in a surrounding area of $40^{\circ} \times 40^{\circ}$. While the low-low tracking concept shows the typical stripes caused by the North-South observation direction that are known from the GRACE temporal gravity models, the MOBILE concept exhibits a quite isotropic error structure at both latitudes, which is quite close to the ideal isotropic signature of concentric rings around the first point.
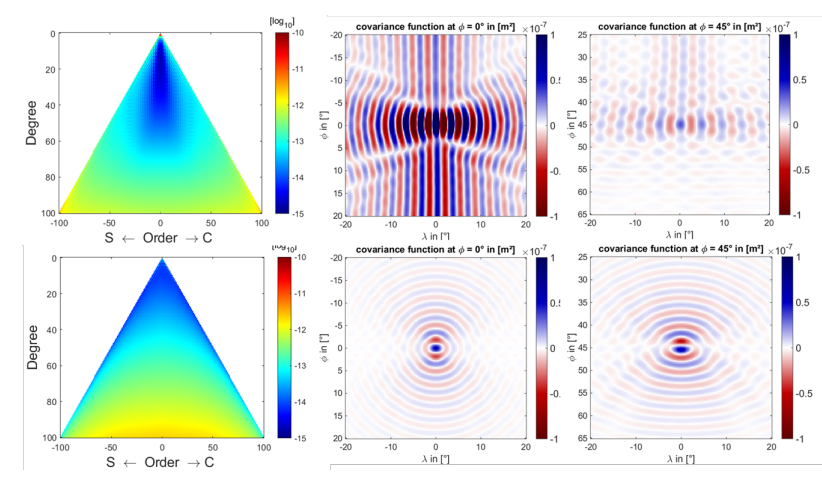

Fig. 4. Formal error triangle plots in $\log _{10}$ (left), and spatial covariance functions (middle column for equator, right column for $45^{\circ}$ latitude) for GRACE type (top row), and MOBILE constellation (bottom row). 


\subsection{Key payload}

The main instrument of MOBILE is a laser-based distance or distance change measurement system, which will be placed at the LEO. Measurement of inter-satellite ranges by laser range interferometry (LRI) has been established for the GRACE-FO mission. In this case, the link between the two satellites was generated with an active laser on one satellite, and a phase-locked amplifying transponder on the second spacecraft. For the MOBILE mission concept the instrument logic needs to be adapted in two ways. Firstly, there will be very large nominal changes in the distance and relative speed, leading to a range of Doppler shifts of several GHz vs. the few MHz observed on GRACEFO. Thus a reference laser source with a larger range of reference frequencies and a faster phase-tracking capability than implemented for GRACE-FO is required. The required parameters $(<10 \mathrm{GHz}$ range, $<10 \mathrm{MHz} / \mathrm{s}$ tracking) are within the range of existing, space qualified reference lasers (e.g., the one used for the ATLID instrument on the EarthCARE mission), but their compatibility with the needs of an interferometric instrument has to be subject of further studies.

Secondly, the link implementation has to be modified as the relative motion of the LEO and MEO satellites also requires pointing tracking capabilities. The impact on overall mission complexity and cost is minimised if a tracking mechanism is used on one partner only (at LEO), while the partner satellites (MEOs) are equipped with passive retroreflectors. Laser tracking and ranging has been successfully performed for decades with active laser systems on ground and passive retroreflectors on satellites in orbit (e.g., LAGEOS, BLITS). The scientific benefit of deploying a passive payload in space is the significantly increased mission duration when compared to complex active payloads. The main technological challenge in utilising this setup for an LRI instrument is the need to achieve a sufficiently high level of received power without the need of amplification between the two passes, ideally close to the $80 \mathrm{pW}$ received by the GRACE-FO implementation, but at least to levels above $\sim 1 \mathrm{pW}$ in order to allow phase tracking. The main design factors impacting the received power are the initial output power, the size of the retroreflector, and the size of the receiving telescope.

For the measurement of non-gravitational forces all satellites are equipped with accelerometers (ACC), which are placed in the center of mass of the satellites. A certain level of compensation of non-conservative accelerations in along-track direction is required for the LEO satellite in order to remove the major part of the disturbing signal, and thus to reduce the measurement range. For MO-
BILE we seek for a resolution on the level of $10^{-11} \mathrm{~m} / \mathrm{s}^{2}$, ideally with the same performance in all three directions. In case only 2 out of 3 axes are high-sensitive, they should be oriented in flight direction (largest signal) and in radial direction following the concept of the GRACE-FO design.

The implementation of an accelerometer is absolutely necessary on the LEO. Based on the heritage of previous gravity missions an electrostatic accelerometer with a measurement bandwidth of 1 to $50 \mathrm{mHz}$ shall be implemented. Less stringent requirements might apply for the MEO satellites, because of the substantially smaller amplitude of the signal and the fact, that non-conservative forces can be modelled much more accurately in high altitudes. To support this modelling, also the design of the MEO could be optimised. As an example, by implementing very simple geometrical surfaces, non-gravitational forces related to radiative pressure acting on the satellite could be modelled quite accurately instead of actually measuring them. In spite of these facts, in the MOBILE concept also for the MEO satellites the implementation of accelerometers was proposed, but technological options could be employed for (one of) the MEOs by replacing the electrostatic instrument by innovative technologies. They include:

- Cold atom or hybrid accelerometer: The applicability of cold atom interferometers (CAI) for space-borne gravimetry has been outlined by Carraz et al. (2014). ONERA is currently developing a hybrid instrument by combining electrostatic and CAI accelerometers (Zahzam et al. 2017). The specific assets of electrostatic sensors, such as their low noise level in a selected measurement bandwidth and their space maturity, are complemented by the CAI's white noise spectral behaviour, which is especially beneficial for gravity recovery in the low-frequency range, and the absolute nature of measurement, thus avoiding the need for calibration.

- Opto-mechanical accelerometer: Novel optomechanical technologies are currently being pioneered by the Institute of Space Systems of the German Aerospace Center (DLR) in collaboration with the Chair of Space Technology at the University of Bremen. These technologies consist of monolithic parallelogram mechanical oscillators of extremely low loss which are integrated with micro-optical displacement sensors that monitor the test mass displacement (Guzmán et al. 2014), yielding highly compact and light weight opto-mechanical inertial sensors of high sensitivity.

- MEMS sensors: Micro-accelerometers based on micro electromechanical system (MEMS) technology, as they are developed by the Imperial College London, have 
the potential to reach noise levels of $10^{-11} \mathrm{~m} / \mathrm{s}^{2} / \sqrt{ } \mathrm{Hz}$. MEMS technology offers the potential to miniaturize the instrument and reduce the weight (currently down to about $600 \mathrm{~g}$ ). Further attractive features are 3D capabilities, and their use as an angular accelerometer supporting attitude determination (Liu and Pike 2016).

All satellites shall be equipped with GNSS space receivers. Geo-location of satellite observations as well as gravity field retrieval require highly accurate continuous orbit determination. Absolute kinematic positioning for geolocation shall be on the $\mathrm{cm}$ level. Using a laser ranging instrument as main measurement system requires exact pointing of the tracking antenna in the order of $10 \mu \mathrm{rad}$ or less, and therefore the implementation of systems for attitude determination and control. The MEO satellites will very likely require an electrical propulsion system to move to their target orbit from the lower separation altitude achievable with a low-cost launcher.

\section{Numerical simulation and results}

The science requirements of the MOBILE mission have been derived in Section 2.2, and are summarized in Table 1. In order to evaluate if these science requirements can be achieved by the mission constellation described in Section 3.1, numerical closed-loop simulations including the main error sources in terms of realistic noise assumptions have been performed. The simulation approach is described in detail by Hauk et al. (2017).

The following orbit parameters have been chosen: The MEOs orbit in an altitude of $10798 \mathrm{~km}$ and have a repeat period of 84 days, while the LEO orbits in an altitude of $346 \mathrm{~km}$ with a repeat period of 28 days. All orbits are polar to avoid relative orbit drifts. The MEOs are in the same orbital plane, separated by 180 -degree mean anomaly for the minimum configuration of $2 \mathrm{MEO}$, and 120/90 degrees in case of 3 or 4 MEOs, respectively.

Regarding errors of the key instruments, the error specifications as outlined in Chapter 3 have been used, i.e. a $1 \mu \mathrm{m}$ noise level of the laser ranging instrument, as well as a noise level of $10^{-11} \mathrm{~m} / \mathrm{s}^{2} / \sqrt{ } \mathrm{Hz}$ in the measurement bandwidth for the accelerometer. In order to simulate geophysical signals, ESA's Earth System Model (Dobslaw et al. 2014) has been used, which contains the five main geophysical signal components atmosphere (A), oceans (O), hydrology (H), ice (I) and solid Earth (S), together abbreviated AOHIS, with a time resolution of 6 hours. Optionally, also ocean tide errors, represented by a difference between two independent ocean tide models, can be included in the simulation.

On top of the instrument errors also temporal aliasing from high-frequency geophysical signals is a main error contributor. It can be significantly reduced by enhanced parameterization strategies such as "Wiese" parameterization by co-estimation of independent daily global gravity fields with reduced spatial resolution corresponding to a maximum spherical harmonic degree and order between 10 and 20, depending on the constellation (Wiese et al. 2011). In contrast, due to their known excitation periods, explicit ocean tide parameters can be co-estimated as well (Hauk and Pail 2018). Several simulation runs have been performed, by switching on and off the different error sources.

At first it is investigated if the observation requirements of the core instruments (LRI and ACC) are capable to meet the science objectives for the chosen orbit (mainly driven by the orbit altitude). For this task, simulations including only instrument errors were performed. Gravity field solutions have been performed for the 28-day period, which coincides with the repeat period of the LEO. Figure 5 shows the results in terms of EWH errors per degree, and Fig. 6 for cumulative EWH errors. The threshold science requirements as specified in Table 1 are indicated in orange colour for $400 \mathrm{~km}$ (corresponding to SH degree 50) and $200 \mathrm{~km}$ (SH degree 100), clearly demonstrating that a mission with the specified instrument performance, together with a LEO orbit altitude of about $350 \mathrm{~km}$, is safely below the threshold requirements even for the minimum configuration of 2 MEOs and 1 LEO (blue curve). Further improved mission performance can be achieved by the extended configurations $3 \mathrm{MEOs/1} \mathrm{LEO} \mathrm{(green)} \mathrm{and} 2 \mathrm{MEOs/2}$ LEOs (magenta). As a reference, the expected performance of the GRACE-FO mission, assuming an inter-satellite ranging accuracy of $50 \mathrm{~nm}$ and the same performance of the accelerometers as for the MOBILE case, is shown in red. Evidently, even though a higher inter-satellite ranging performance of GRACE-FO was assumed, the MOBILE concept outperforms the GRACE-type mission especially in low degrees due to the superior constellation geometry.

From Fig. 7 one can determine the performance one can achieve with the different mission constellations when including also aliasing errors from hydrology (H), ice (I) and solid Earth (S), together abbreviated HIS, in the simulation. The threshold science requirements are almost achieved for $400 \mathrm{~km}$ spatial resolution (SH degree 50); in fact, the $5 \mathrm{~mm}$ EHW is reached at degrees 43 to 46 for the different high-low scenarios. For $200 \mathrm{~km}$ (SH degree 100) the required $10 \mathrm{~cm}$ EWH level is crossed at degree 


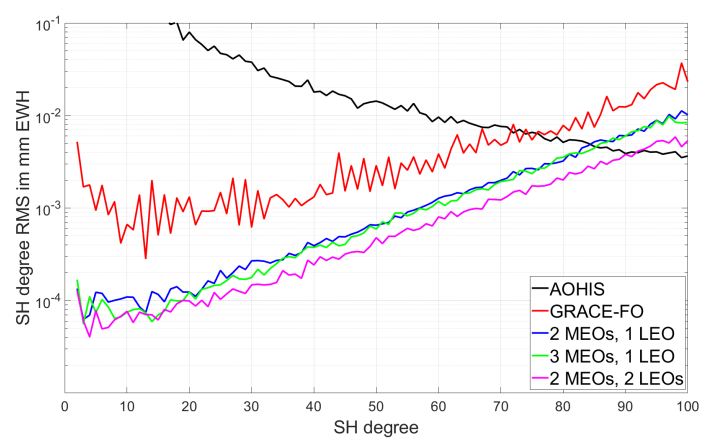

Fig. 5. Degree (error) RMS of full AOHIS signal (black) and different mission scenarios, when including only instrument errors.

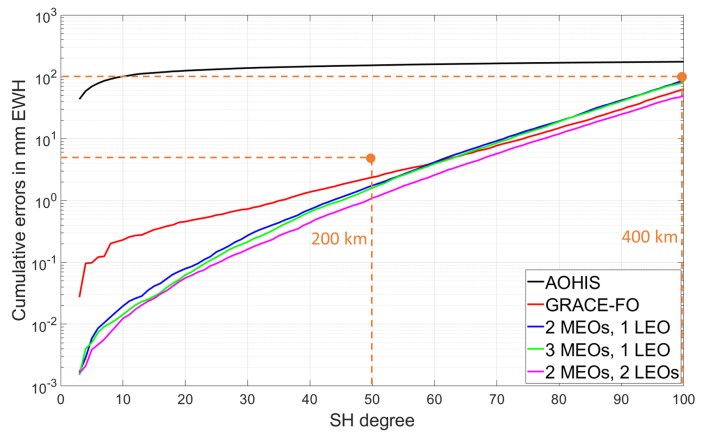

Fig. 6. Cumulative signal of full AOHIS (black) and cumulative errors of different mission scenarios, when including only instrument errors. The threshold science requirements for $400 \mathrm{~km}$ (SH degree $50)$ and $200 \mathrm{~km}$ (SH degree 100) are included in orange colour.

96 for the configuration 2 MEOs/1 LEO. As a reference, a GRACE-FO scenario is included (red curve), showing that MOBILE, even in the minimum configuration, will outperform GRACE-FO by a factor of 5 in terms of mass transport observations.

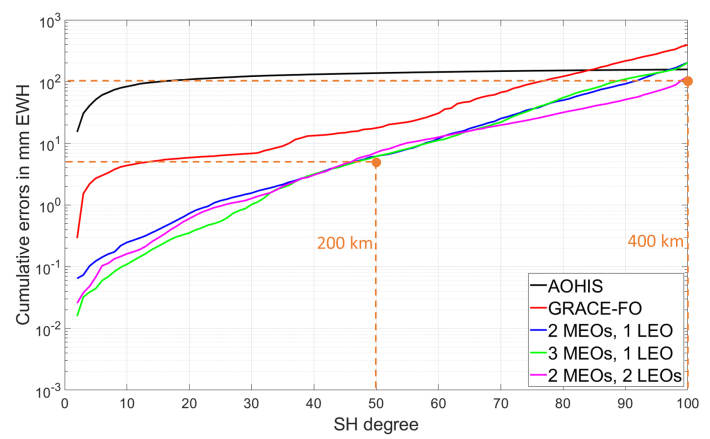

Fig. 7. Cumulative signals/errors when including instrument errors and aliasing errors from hydrology, ice and solid Earth (HIS). The threshold science requirements for $400 \mathrm{~km}$ (SH degree 50) and $200 \mathrm{~km}$ (SH degree 100) are included in orange colour.
In Fig. 8 the differences of the monthly true mass transport model and the recovered signal are shown in spatial domain for the MOBILE minimum configuration, and the GRACE-FO case as a reference. Both cases are resolved up to SH degree 50. As already suggested by Fig. 4, the error pattern of MOBILE is much more homogeneous, and the typical striping of a low-low along-track ranging system is significantly reduced.

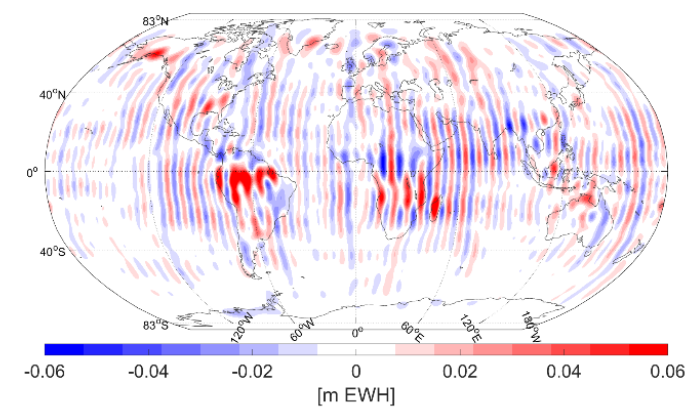

(a)

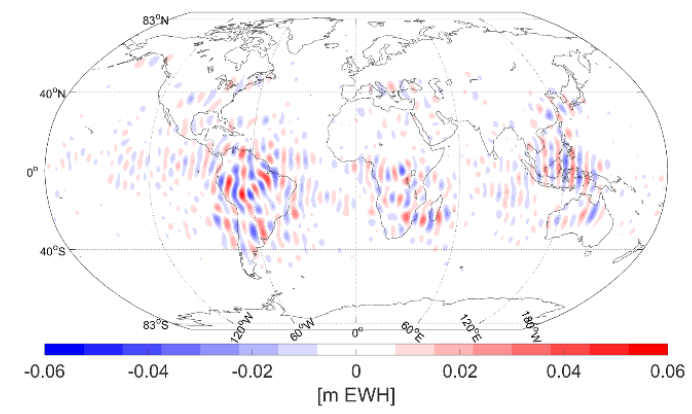

(b)

Fig. 8. Global grids of EWH [m] up to d/o 50 for GRACE (a) and MOBILE minimum configuration (b).

The application of extended parameterization schemes such as the Wiese approach as discussed above opens the possibility to estimate short period mass transport signals together with the targeted monthly solutions. In this respect, an interesting question to be analysed is how much signal can be represented by the 2-daily mass transport parameters, which have been co-estimated up to SH degree 10 in the frame of the gravity field retrieval. In this case the full AOHIS signal was used as an input and also retrieved in the simulation. Figure 9 shows degree RMS curves of the full mass transport signal (black curves) and the deviations to the estimated 2-daily retrieved gravity fields (blue curves) of all 2-day periods within the 28-day orbit period. Evidently the 2-daily gravity field can 
be estimated with a signal-to-noise ratio of almost one order of magnitude. They reflect mainly short-periodic $\mathrm{AO}$ signals, but also high-frequency hydrology signals (Daras and Pail 2017).

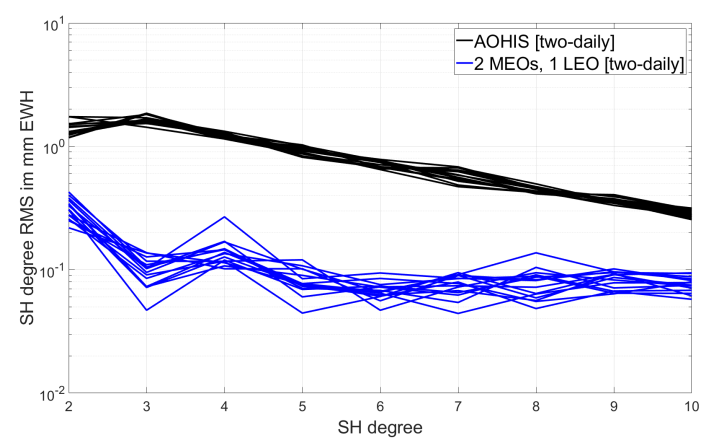

Fig. 9. Degree RMS of 2-daily gravity field solutions resolved up to $\mathrm{SH}$ degree 10. The full AOHIS signal is included in the observations, and recovered in the retrieval process. For details see text.

Recapitulating the simulation results one can conclude that the achievable performance of MOBILE is in agreement with the threshold science requirements, which can be even slightly exceeded with an extended configuration. It should be emphasized that for all simulation results that have been shown here there is a significant potential of further optimization regarding (a) the orbit configuration, and (b) the processing schemes, which will have to be further investigated in more depth for the high-low scenario. First numerical closed-loop simulations applying a full-scale simulation tool (Daras et al. 2015) largely confirm the results presented here. They will be part of future work.

\section{Conclusions and outlook}

The main conclusion from the numerical closed-loop simulations is, that even with the minimum configuration of 1 LEO and 2 MEOs the threshold science requirements can be met by MOBILE. Compared to a GRACE-FO scenario, the resulting gravity field performance of MOBILE improves by a factor of about 5 , especially in the long wavelengths where the largest amplitudes of time-variable gravity field signals occur. Regarding a substantial improvement in temporal resolution, an extension of the minimum configuration, especially the implementation of more than one LEO, would be required.

In Chapter 1 it was already pointed out, that according to GCOS recommendations at least 30 years of obser- vations are needed to separate human-induced from natural climate variation processes and to estimate trends with high significance. This recommendation triggers a great need of the international user communities for sustained observation of mass transport from space, requiring observation concepts which can be implemented on an operational basis. They could be implemented as future element of the Copernicus/Sentinel programme, and making benefit of already existing European EO infrastructure. The proposed MOBILE configuration can be regarded as a precursor and role model and pioneering concept for a future sustained mass transport observing system from space at reasonable cost level. As such, it would significantly advance European EO capabilities with a central observation type, addressing several ECVs.

Due to its modularity, the proposed mass transport mission can be maintained and even further expanded by additional elements. Due to their largely passive instrumentation, the function of the MEO satellites could be implemented as backpack application of other MEO missions, such as the Galileo next-generation satellites, in order to extend and maintain the infrastructure for laser ranging payloads. Alternatively, dedicated MEO satellites carry the potential of miniaturization. Adding satellites or co-using existing infrastructure would enable us to achieve significantly higher spatial and temporal resolution than with the minimum configuration. With such a future concept there is a realistic opportunity to tackle even the target science requirements.

The MEO satellites could also serve as platforms for additional payload. As an example, elements of the European Reference Station in Space (E-GRASP; Biancale et al. 2017) concept could be included as a technology demonstrator on the MEO(s), because the MOBILE MEO orbit is well suited for achieving most of the E-GRASP objectives. Additional payload could consist of a VLBI transmitter a time transfer by laser link (T2L2) instrument, and a highprecision clock. Of course additional payload on the MEO would increase their complexity and costs.

In spite of the "high scientific merit" that was emphasized in ESA's evaluation report, unfortunately the MOBILE proposal was not selected as one out of three candidates of an Earth Explorer 10 mission due to "technical and programmatic risks". Still the authors believe that MOBILE is an interesting concept for sustained mass transport monitoring from space to be pursued in the future. 


\section{References}

Bender P.L., Wiese D.N. and Nerem R.S., 2008, A possible DualGRACE mission with 90 degree and 63 degree inclination orbits, Proceedings of the 3rd International Symposium on Formation Flying, Missions and Technologies. European Space Agency Symposium Proceedings, SP-654 jILA Pub. 8161, Noordwijk, Netherlands

Biancale R. et al., 2017, E-GRASP/Eratosthenes, Proposal for Earth Explorer Opportunity Mission EE-9 in response to the Call for Proposals for Earth Explorer Opportunity Mission EE-9 (ESA/EXPLORER/EE-9 rev. 1), June 2017

Carraz O., Siemes, C. Massotti L., Haagmans R. and Silvestrin P., 2014, A Spaceborne Gravity Gradiometer Concept Based on Cold Atom Interferometers for Measuring Earth's Gravity Field, Microgravity Science and Technology, 26(3), 139-145, DOI: https://doi.org/10.1007/s12217-014-9385-x

Daras I. and Pail R., 2017, Treatment of temporal aliasing effects in the context of next generation satellite gravimetry missions, J. Geophys. Res: Solid Earth, 122(9), 7343-7362, DOI: 10.1002/2017JB014250

Daras I., Pail R., Murböck M. and Yi W., 2015, Gravity field processing with enhanced numerical precision for LL-SST missions, J. Geod., 89, 99-110, DOI: 10.1007/s00190-014-0764-2

Dobslaw H., Bergmann-Wolf I., Dill R., Forootan E., Klemann V., Kusche J. and Sasgen I., 2014, Updating ESA's Earth System Model for Gravity Mission Simulation Studies: 1. Model Description and Validation, GFZ, 69 p., DOI: http://doi.org/10. 2312/GFZ.b103-14079

Drinkwater M.R., Floberghagen R., Haagmans R., Muzi D. and Popescu A., 2003, GOCE: ESA's first Earth Explorer Core mission, In: G. Beutler et al. (Eds.): Earth Gravity Field from Space - from Sensors to Earth Science, Space Sciences Series of ISSI (Vol. 18). Dordrecht, Netherlands, 419-432, ISBN:1-40201408-2

Flechtner, F., Neumayer, K.H., Dahle, C., Dobslaw, H., Güntner, A., Raimondo, J.C. et al., 2016, What can be expected from the GRACE-FO Laser Ranging Interferometer for Earth Science applications?, Surv. Geophys., 37(2), 453-470, DOI: 10.1007/s10712-015-9338-y

GCOS, 2016, The Global Observing System for Climate: Implementation Plan. Global Climate Observing System, World Meteorological Organization, GCOS-200 (GOOS-214),, https: //unfccc.int/sites/default/files/gcos_ip_10oct2016.pdf

Gruber T., Panet I., e.motion2 Team, 2015, Proposal to ESA's Earth Explorer Call 9: Earth System Mass Transport Mission - e.motion2, Deutsche Geodätische Kommission der Bayerischen Akademie der Wissenschaften, Reihe B, Angewandte Geodäsie, Series B, https://dgk.badw.de/fileadmin/user_ upload/Files/DGK/docs/b-318.pdf

Guzmán Cervantes F., Kumanchik L., Pratt J. and Taylor J.M., 2014, High sensitivity optomechanical reference accelerometer over 10 kHz, Appl. Phys. Lett., 104, 221111, DOI: https://doi.org/10. 1063/1.4881936

Hauk M. and Pail R., 2018, Treatment of Ocean Tide Aliasing in the context of a Next Generation Gravity Field Mission, Geophy. J. Int., 214(1), 345-365, DOI: https://doi.org/10.1093/gji/ggy145

Hauk M., Schlicht A., Pail R. and Murböck M., 2017, Gravity field recovery in the framework of a Geodesy and Time Reference in
Space (GETRIS), Adv. Space Res., 59(8), 2032-47, DOI: https: //doi.org/10.1016/j.asr.2017.01.028

IGSWG, NASA/ESA Interagency Gravity Science Working Group, 2016, Towards a sustained observing system for mass transport to understand global change and to benefit society,, Doc. Nr.: TUD-IGSWG-2016-01

IUGG, International Union of Geodesy and Geophysics, 2015, Resolutions adopted by the council at the XXVI General Assembly Prague, Czech Republic,, 22. June to 2. July 2015

Liu H. and Pike W.T., 2016, A micromachined angular-acceleration sensor for geophysical applications, Appl. Phys. Lett., 109(17), 173506, DOI: 10.1063/1.4966547

Murböck M., Pail R., Daras I. and Gruber T., 2014, Optimal orbits for temporal gravity recovery regarding temporal aliasing, J. Geod., 88(2), 113-126, DOI: 10.1007/s00190-013-0671-y

Murböck M., Pail R., Daras I. and Gruber T., 2014, Optimal orbits for temporal gravity recovery regarding temporal aliasing, J. Geod., 88(2), 113-126, DOI: 10.1007/s00190-013-0671-y

NAS - National Academies of Sciences, Engineering, and Medicine, 2018, Thriving on Our Changing Planet: A Decadal Strategy for Earth Observation from Space, Washington, DC: The National Academies Press, https://essp.nasa.gov/essp/files/2018/02/ 2017-Earth-Science-Decadal-Survey.pdf

Pail R. et al., 2015, Observing mass transport to understand global change and benefit society: science and user needs. An international multi-disciplinary initiative for IUGG, Edited by Roland Pail, Deutsche Geodätische Kommission der Bayerischen Akademie der Wissenschaften, Heft 320, ISBN 978-3-76968599-2, München, https://www.dgk.badw.de/fileadmin/user_ upload/Files/DGK/docs/b-320.pdf

Pail R., Bingham R., Braitenberg C., Dobslaw H., Eicker A., Güntner A. et al., 2015, IUGG Expert Panel, Science and user needs for observing global mass transport to understand global change and benefit society, Surv. Geophys., 36(6), 743-772, DOI: $10.1007 /$ s10712-015-9348-9

Panet I., Flury J., Biancale R., Gruber T., Johannessen J., van den Broeke M., et al., 2012, Earth System Mass Transport Mission (e.motion): A Concept for future Earth Gravity Field Measurements from Space, Surv. Geophys., 34(2), 141-163, DOI: 10.1007/s10712-012-9209-8

Tapley B., Bettadpur S., Watkins M. and Reigber C., 2004, The gravity recovery and climate experiment: Mission overview and early results, Geophys. Res. Lett., 31(9), DOI: 10.1029/2004GL019920

Wiese D.N., Visser P.N.A.M. and Nerem R.S., 2011, Estimating low resolution gravity fields at short time intervals to reduce temporal aliasing errors, Adv. Space Res., 48, 1094-1107, DOI: 10.1016/j.asr.2011.05.027

Zahzam N., Bidel Y., Bresson A., Huynh P.-A., Liorzou F., Lebat V., et al., 2017, Hybrid Atom Electrostatic System for Satellite Geodesy, Geophysical Research Abstracts, 19, EGU2017-149581, EGU General Assembly 2017 